\title{
Electrochemical, morphological and microstructural characterization of carbon film resistor electrodes for application in electrochemical sensors
}

\author{
Carla Gouveia-Caridade $^{\mathrm{a}}$, David M. Soares ${ }^{\mathrm{b}}$, Hans-Dieter Liess ${ }^{\mathrm{c}}$, Christopher M.A. Brett $^{\mathrm{a}, *}$ \\ a Departamento de Química, Faculdade de Ciências e Tecnologia, Universidade de Coimbra, 3004-535 Coimbra, Portugal \\ ${ }^{\mathrm{b}}$ Instituto de Física Gleb Wataghin, Unicamp, Campinas, SP, Brazil \\ ' Institut für Physik, Fakultät für Elektrotechnik, Universität der Bundeswehr München, D-85577 Neubiberg, Germany
}

\section{A R T I C L E IN F O}

\section{Article history:}

Received 7 February 2008

Received in revised form 31 March 2008

Accepted 31 March 2008

Available online $\mathrm{xxx}$

\section{Keywords:}

Carbon film electrodes

Carbon film resistors

Cyclic voltammetry

Electrochemical impedance spectroscopy

Contact mode AFM

Confocal Raman spectroscopy

$\mathrm{X}$-ray diffraction

\begin{abstract}
A B S T R A C T
The electrochemical and microstructural properties of carbon film electrodes made from carbon film electrical resistors of $1.5,15,140 \Omega$ and $2.0 \mathrm{k} \Omega$ nominal resistance have been investigated before and after electrochemical pre-treatment at $+0.9 \mathrm{~V}$ vs SCE, in order to assess the potential use of these carbon film electrodes as electrochemical sensors and as substrates for sensors and biosensors. The results obtained are compared with those at electrodes made from previously investigated $2 \Omega$ carbon film resistors. Cyclic voltammetry was performed in acetate buffer and phosphate buffer saline electrolytes and the kinetic parameters of the model redox system $\mathrm{Fe}(\mathrm{CN})_{6}{ }^{3-14-}$ obtained. The $1.5 \Omega$ resistor electrodes show the best properties for sensor development with wide potential windows, similar electrochemical behaviour to those of $2 \Omega$ and close-to-reversible kinetic parameters after electrochemical pre-treatment. The 15 and $140 \Omega$ resistor electrodes show wide potential windows although with slower kinetics, whereas the $2.0 \mathrm{k} \Omega$ resistor electrodes show poor cyclic voltammetric profiles even after pre-treatment. Electrochemical impedance spectroscopy related these findings to the interfacial properties of the electrodes. Microstructural and morphological studies were carried out using contact mode Atomic Force Microscopy (AFM), Confocal Raman spectroscopy and X-ray diffraction. AFM showed more homogeneity of the films with lower nominal resistances, related to better electrochemical characteristics. X-ray diffraction and Confocal Raman spectroscopy indicate the existence of a graphitic structure in the carbon films.
\end{abstract}

(c) 2008 Elsevier B.V. All rights reserved.

\section{Introduction}

New electrode materials have recently been studied for environmental and health applications, particularly to avoid the traditionally used mercury. For application as electrochemical sensors, such materials must have a large potential window and low background current, as well as simple surface regeneration and inertness [1].

Carbon is a commonly used solid electrode material, particularly in the form of glassy carbon, due to its wide positive potential window, mechanical stability, and low porosity. Carbon film electrodes made from carbon film resistors, obtained by coating a substrate with a thin pyrolytic carbon layer, have recently been introduced as a promising alternative form of carbon electrode. Carbon film resistor electrodes have a large potential window after electrochemical surface pre-treatment [2] and have been char-

\footnotetext{
* Corresponding author. Tel.: +351 239 835295; fax: +351 239835295.

E-mail address: brett@ci.uc.pt (Christopher M.A. Brett).
}

acterized [2-4] and successfully applied to the development of sensors, e.g. [4-9] and biosensors, e.g. [10-13].

Atomic Force Microscopy (AFM) is a well-established technique which has been used in many applications and in the study of the surface morphology of materials, including thin and thick coatings, ceramics, composites, glasses, synthetic and biological membranes, metals, polymers, and semiconductors [14].

Raman spectroscopy allows the characterization of the molecular or chemical composition of many types of samples without any specific preparation, and is a rapid and nondestructive technique and is a very effective way to investigate the detailed bonding structure of carbon films [14]. Confocal Raman spectroscopy is a specific form of Raman spectroscopy that can be used where a high spatial resolution is required. It has been widely applied in several fields, imaging microscopy being one of the most extensive applications, because it provides 3D spatial resolution. The major advantage of confocal Raman spectroscopy is the ability to collect Raman scatter from a small point within the interior of a larger sample. Typical applications are in structure determination, multicomponent qualitative and quantitative 
analysis. In materials science it has been used for sample composition and crystallinity studies [15].

The purpose of this work was to evaluate the electrochemical and microstructural characteristics of carbon film electrical resistors of different resistances, from $1.5 \Omega$ to $2.0 \mathrm{k} \Omega$, as electrodes for sensor and biosensor applications with and without pre-treatment. Comparison is made with results obtained at previously investigated carbon film electrodes of $2 \Omega$ nominal resistance [2-4].

\section{Experimental}

\subsection{Electrode preparation}

Electrodes were made from carbon film electrical resistors of different nominal values: $1.5,2.0,15,140 \Omega$ and $2.0 \mathrm{k} \Omega$; the preparation protocol is described in detail elsewhere [3]. The preparation of the carbon film resistors by pyrolysis of hydrocarbons on ceramic cylinder substrates inside a quartz bottle held in a furnace at $1100{ }^{\circ} \mathrm{C}$ in a continuous flow of nitrogen containing a fraction of hydrocarbons (usually methane) is described in greater detail in [2]. The cylindrical resistors used have length $0.6 \mathrm{~cm}$ and external diameter $0.15 \mathrm{~cm}$, with two tight-fitting metal caps and connecting wires as external contact. Briefly, to prepare the electrodes one of these metal caps is removed and after sheathing the connecting wire, the other is protected by normal epoxy resin. The final exposed carbon film electrode geometric area is $\sim 0.20 \mathrm{~cm}^{2}$.

Nafion coatings, when required, were made by placing $5 \mu \mathrm{L}$ of a solution of $0.25 \mathrm{wt} \%$ Nafion in alcohols, followed by $3 \mu \mathrm{L}$ of $N, N^{\prime}$-dimethylformamide, directly on top of the surface of the carbon film electrode. After evaporation of the solvents, the film was cured with a jet of warm air for about $1 \mathrm{~min}$. The film is of ca. $1 \mu \mathrm{m}$ thickness. This procedure was the same as used in [16].

\subsection{Instrumentation and methods}

Voltammetric experiments and Electrochemical Impedance Spectroscopy (EIS) were carried out using a standard threeelectrode configuration in a glass cell of capacity $15 \mathrm{~cm}^{3}$. Besides the carbon film working electrode, the cell contained a platinum wire counter electrode and a saturated calomel electrode (SCE) as reference. Electrochemical pre-treatment of the electrodes, was done by applying a potential of $+0.9 \mathrm{~V}$ vs SCE during periods of $1-$ $6 \mathrm{~min}$ in the buffer electrolyte solution in which experiments were conducted.

Voltammetric experiments were conducted using a $\mu$ Autolab II potentiostat (Eco Chemie, Utrecht, Netherlands) controlled by GPES 4.9 software.

Electrochemical impedance spectra were recorded using a Solartron 1250 Frequency Response Analyzer coupled to a Solartron 1286 Electrochemical Interface using ZPlot 2.4 software (Solartron Analytical, UK). The frequency range from $65.5 \mathrm{kHz}$ to $0.1 \mathrm{~Hz}$ was scanned logarithmically with an applied sinusoidal voltage perturbation of amplitude $10 \mathrm{mV}, 10$ points per frequency decade, superimposed on the chosen applied potential. An autointegration time of $60 \mathrm{~s}$ was employed.

AFM was performed with a Multimode ${ }^{\mathrm{TM}}$ Atomic Force Microscope controlled by a Digital Instruments Nanoscope E controller (Veeco Instruments, USA). Silicon nitride NanoProbes ${ }^{\mathrm{TM}} \mathrm{V}$-shaped cantilevers, $100 \mu \mathrm{m}$ length, $0.58 \mathrm{~N} \mathrm{~m}^{-1}$ spring constant were used. All images were recorded in contact mode AFM in air at room temperature. Image contrast and brightness were adjusted.
Confocal Raman spectroscopy was performed using a Scanning Near-field Optical Microscope, AlphaSNOM (WiTec, Germany).

The microstructure was evaluated by X-ray diffraction (XRD). The diffraction patterns were acquired from a Philips X'Pert apparatus (PANalytical, Netherlands) with a Co anode $\left(\lambda_{k \alpha 1}=1.78896 \AA, \lambda_{k \alpha 2}=1.79285 \AA\right)$ and a collimated detector in Bragg-Brentano mode.

\subsection{Reagents and solutions}

All solutions were made from analytical grade reagents and MilliQ ultrapure water (resistivity $\geq 18 \mathrm{M} \Omega \mathrm{cm}$ ). Solutions used as supporting electrolytes were $0.1 \mathrm{M}$ phosphate buffer saline solution (PBS), pH 7.0, prepared from sodium dihydrogenphosphate, disodium hydrogenphosphate and $0.05 \mathrm{M} \mathrm{NaCl} ; 0.1 \mathrm{M}$ sodium acetate/acetic acid buffer, $\mathrm{pH} 4.2$, and $0.4 \mathrm{M}$ potassium sulphate. All experiments were carried out at room temperature $\left(25 \pm 1{ }^{\circ} \mathrm{C}\right)$.

\section{Results and discussion}

The electrochemical characterization of the carbon film electrodes was done by cyclic voltammetry and electrochemical impedance spectroscopy. Morphological and microstructural studies were carried out by Atomic Force Microscopy, Confocal Raman spectroscopy and X-ray diffraction. The principal results will be discussed below.
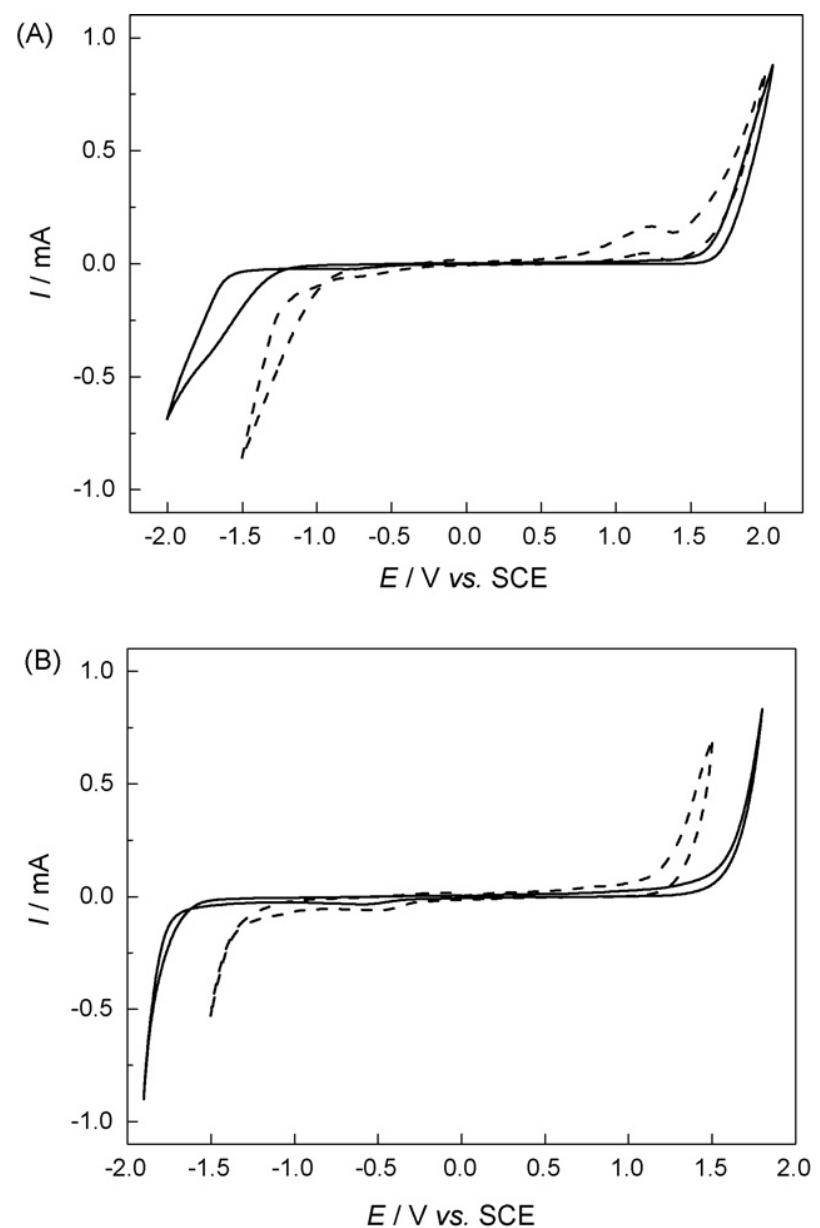

Fig. 1. Cyclic voltammograms in (A) $\mathrm{pH} 4.2$ acetate buffer solution and (B) phosphate buffer saline solution at carbon film electrodes of $1.5 \Omega$ nominal resistance, area $0.2 \mathrm{~cm}^{2}(--)$ before and (-) after pre-treatment. Pre-treatment: $+0.9 \mathrm{~V}$ vs SCE during $4 \mathrm{~min}$ in the same buffer solution. 


\subsection{Electrochemical characterization of the carbon film electrodes}

\subsubsection{Cyclic voltammetry}

3.1.1.1. Potential window. Cyclic voltammetry was used to investigate the potential window of different electrodes in two buffer solutions: $0.1 \mathrm{M}$ acetate buffer, $\mathrm{pH} 4.2$, and $0.1 \mathrm{M}$ phosphate buffer saline, pH 7.0. Fig. 1 shows typical responses for electrodes of $1.5 \Omega$ in acetate buffer, $\mathrm{pH} 4.2$, and phosphate buffer saline, $\mathrm{pH} 7.0$, before and after pre-treatment at a fixed positive potential of $+0.9 \mathrm{~V}$ vs SCE for $4 \mathrm{~min}$ in the electrolyte solution under study. It was found that after holding the electrode at $+0.9 \mathrm{~V}$ the background currents decreased and the potential window increased. Electrochemical pre-treatment for periods between 1 and 6 min was evaluated but no further improvement for periods longer than $4 \mathrm{~min}$ occurred, so that all subsequent experiments used 4 min pre-treatment time.

Changes in the potential window are significant in phosphate buffer saline, with increases of $300-400 \mathrm{mV}$ in the negative and positive potential limits. In acetate buffer the changes are not so great: the negative limit increases by $200-400 \mathrm{mV}$, and the positive limit by less than $200 \mathrm{mV}$. The fixed potential used for pretreatment leads to the oxidation of hydrocarbon groups present in electrode surface, influencing the characteristics and electrochemical behaviour of the sensor. It also confers better stability to the electrode surface and lower capacitive currents. A similar pretreatment was used in [11], when resistors of $2 \Omega$ nominal resistance where employed as substrate for glucose oxidase-based enzyme biosensors leading to better reproducibility in the detection of the $\mathrm{H}_{2} \mathrm{O}_{2}$ produced.

An exception to this behaviour occurs with electrodes made from $2.0 \mathrm{k} \Omega$ resistors, Fig. 2. In this case, in acetate buffer solution, there were no differences in the voltammograms obtained before and after pre-treatment, the potential window being narrow and not very useful for electroanalysis. In phosphate buffer saline solution the voltammetric profiles obtained were poorly defined.

Fig. 3 shows a comparison between voltammograms at different electrodes. Similar potential windows were obtained in [2,3] with carbon film resistors of $2 \Omega$ and in [3] with carbon film resistors of 2 and $20 \Omega$ nominal resistance.

Repetition of these experiments with different carbon film electrodes of the same nominal resistance demonstrated excellent reproducibility in the voltammograms after pre-treatment.

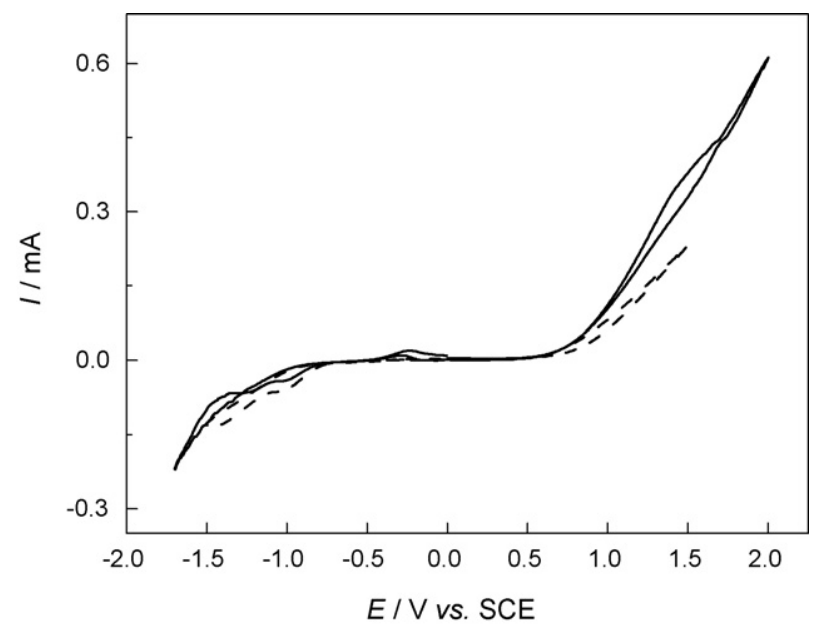

Fig. 2. Cyclic voltammograms in $\mathrm{pH} 4.2$ acetate buffer solution at carbon film electrodes of $2.0 \mathrm{k} \Omega$ nominal resistance, area $0.2 \mathrm{~cm}^{2}(--)$ before and (-) after pretreatment. Pre-treatment: $+0.9 \mathrm{~V}$ vs SCE during $4 \mathrm{~min}$ in the same buffer solution.
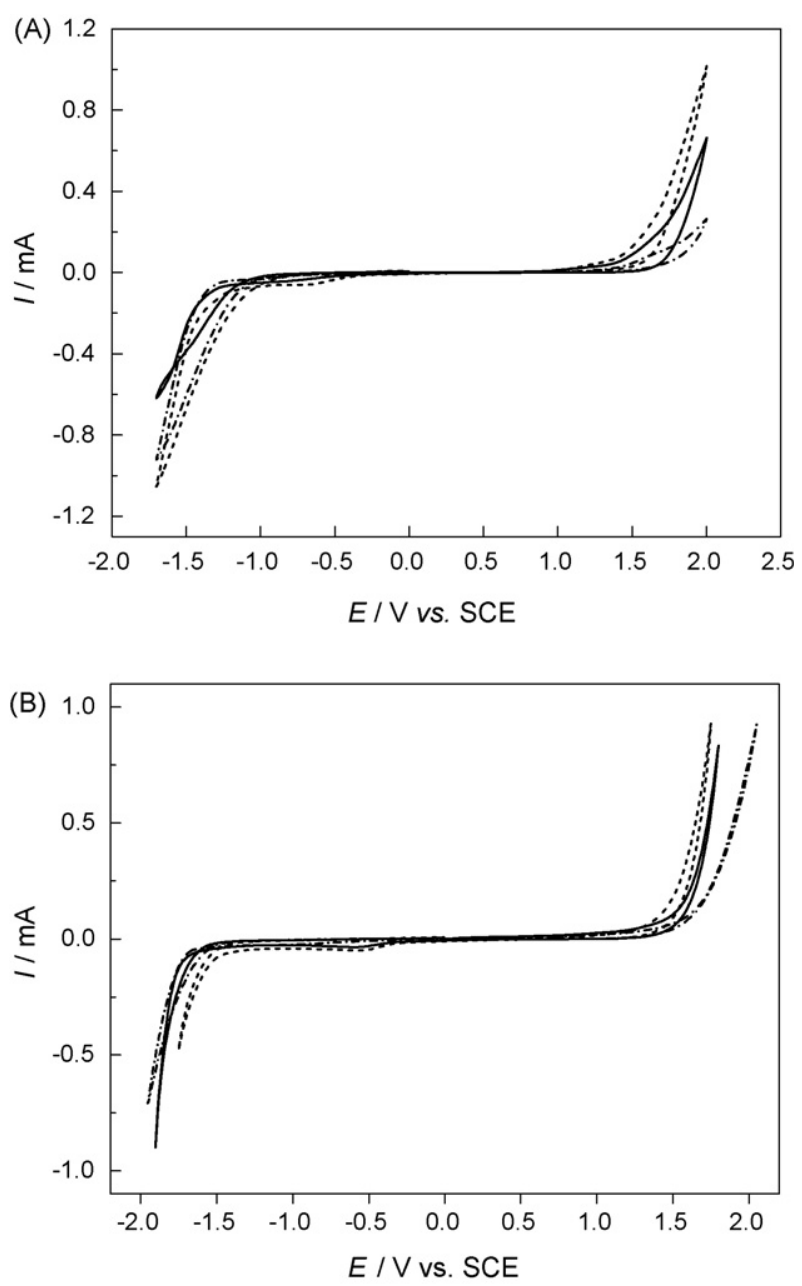

Fig. 3. Cyclic voltammograms in (A) $\mathrm{pH} 4.2$ acetate buffer solution and (B) phosphate buffer saline solution at carbon film electrodes of $(-) 1.5 \Omega,(--) 15 \Omega$ and (- - -) $140 \Omega$ nominal resistances obtained after pre-treatment. Pre-treatment: $+0.9 \mathrm{~V}$ vs SCE during $4 \mathrm{~min}$ in the same buffer solution.

3.1.1.2. Oxidation of potassium hexacyanoferrate(II). Fig. 4 shows typical cyclic voltammograms obtained with carbon film electrodes from $1.5 \Omega$ resistors for the oxidation of hexacyanoferrate(II) in $0.4 \mathrm{M} \mathrm{K}_{2} \mathrm{SO}_{4}$ at different scan rates. These voltammograms were

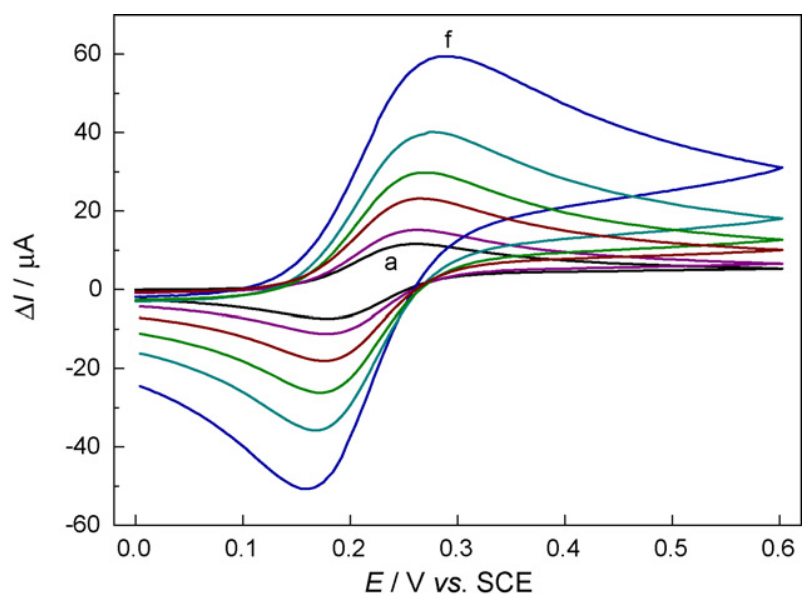

Fig. 4. Cyclic voltammograms obtained with pretreated carbon film electrodes of $1.5 \Omega$ nominal resistance after background subtraction for oxidation of $1 \mathrm{mM}$ $\mathrm{K}_{4} \mathrm{Fe}(\mathrm{CN})_{6}$ in $0.4 \mathrm{M} \mathrm{K}_{2} \mathrm{SO}_{4}$ electrolyte at scan rates of (a) $10 \mathrm{mV} \mathrm{s}^{-1}$ to (f) $500 \mathrm{mV} \mathrm{s}^{-1}$. 
Table 1

Parameters obtained from the cyclic voltammograms in $1 \mathrm{mM} \mathrm{K}{ }_{4} \mathrm{Fe}(\mathrm{CN})_{6}+0.4 \mathrm{M} \mathrm{K}_{2} \mathrm{SO}_{4}$ electrolyte at $1.5 \Omega$ resistor electrodes with and without pre-treatment (PT)

\begin{tabular}{|c|c|c|c|c|c|c|}
\hline \multirow[t]{2}{*}{$v\left(\mathrm{~V} \mathrm{~s}^{-1}\right)$} & \multicolumn{2}{|l|}{$\left|I_{\mathrm{pc}} / I_{\mathrm{pa}}\right|$} & \multicolumn{2}{|l|}{$\Delta E_{\mathrm{p}}(\mathrm{V})$} & \multicolumn{2}{|c|}{$k_{0} \times 10^{3}\left(\mathrm{~cm} \mathrm{~s}^{-1}\right)$} \\
\hline & Without PT & With PT & Without PT & With PT & Without PT & With PT \\
\hline 0.010 & $0.61 \pm 0.01$ & $0.85 \pm 0.01$ & $0.111 \pm 0.005$ & $0.074 \pm 0.002$ & $1.20 \pm 0.14$ & $4.80 \pm 0.65$ \\
\hline 0.020 & $0.62 \pm 0.01$ & $0.88 \pm 0.02$ & $0.120 \pm 0.003$ & $0.075 \pm 0.002$ & $1.42 \pm 0.09$ & $6.31 \pm 0.81$ \\
\hline 0.050 & $0.63 \pm 0.05$ & $0.90 \pm 0.03$ & $0.146 \pm 0.005$ & $0.076 \pm 0.002$ & $1.43 \pm 0.05$ & $9.70 \pm 0.15$ \\
\hline 0.100 & $0.64 \pm 0.03$ & $0.91 \pm 0.04$ & $0.165 \pm 0.005$ & $0.085 \pm 0.003$ & $1.57 \pm 0.03$ & $8.39 \pm 0.13$ \\
\hline 0.200 & - & $0.92 \pm 0.04$ & - & $0.097 \pm 0.003$ & - & $7.55 \pm 0.67$ \\
\hline 0.500 & - & $0.93 \pm 0.05$ & - & $0.108 \pm 0.004$ & - & $9.25 \pm 0.11$ \\
\hline
\end{tabular}

Mean value and standard deviation for three electrodes.

recorded directly after pre-treatment at $+0.9 \mathrm{~V}$ in acetate buffer solution. Voltammograms obtained with electrodes pre-treated in phosphate buffer saline solution were similar to those in Fig. 4.

Table 1 summarises the parameters obtained from the voltammograms for $1.5 \Omega$ resistors before and after pre-treatment. There is an improvement in the kinetics of the electro-oxidation after pre-treatment, with values and trends characteristic of a quasi-reversible system. The standard rate constant, $k_{0}$, was estimated by Nicholson's method from the anodic and cathodic peak potential separation [17], giving values between (4.8 and $9.7) \times 10^{-3} \mathrm{~cm} \mathrm{~s}^{-1}$ for $1.5 \Omega$ electrodes. Similar results were obtained in [2] with carbon film resistors of $2 \Omega$ after pretreatment in perchloric acid, and in good agreement with values at glassy carbon electrodes [18].
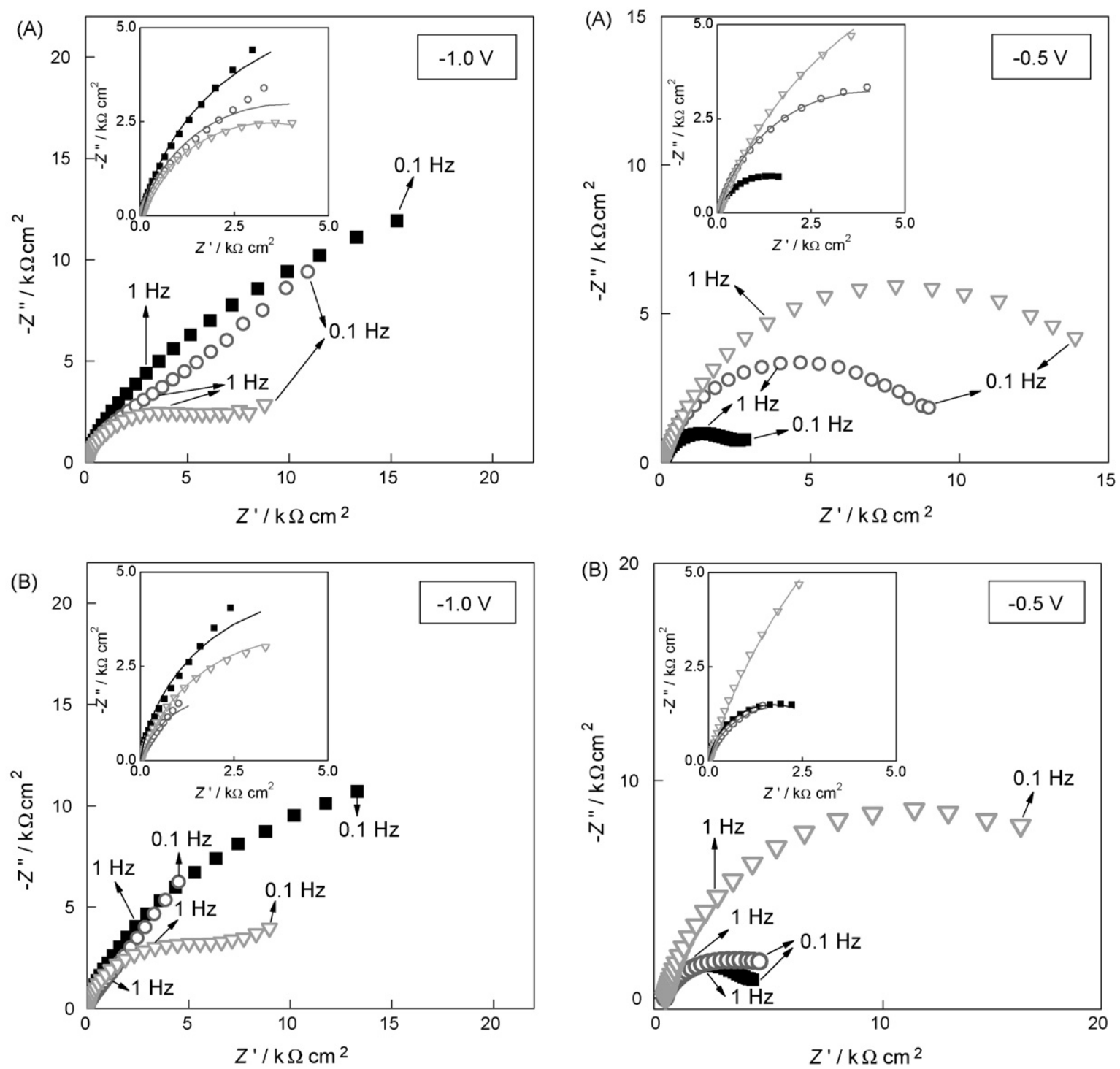

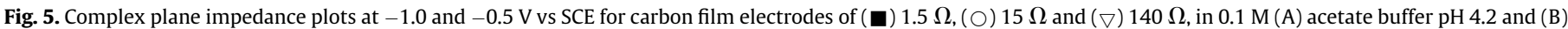

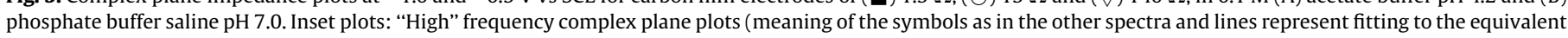
circuit described in the text). 
Table 2

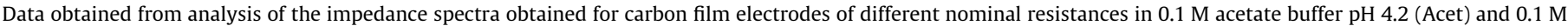
phosphate buffer saline pH 7.0 (PBS)

\begin{tabular}{|c|c|c|c|c|c|c|c|}
\hline \multirow[t]{2}{*}{ Nominal resistance $(\Omega)$} & \multirow[t]{2}{*}{$E(\mathrm{~V}$ vs SCE) } & \multicolumn{2}{|c|}{$R_{1}\left(\mathrm{k} \Omega \mathrm{cm}^{2}\right)$} & \multicolumn{2}{|c|}{$C_{1}\left(\mu \mathrm{F} \mathrm{cm}^{-2} \mathrm{~s}^{\alpha_{-1}}\right)$} & \multicolumn{2}{|l|}{$\alpha$} \\
\hline & & Acet & PBS & Acet & PBS & Acet & PBS \\
\hline \multirow[t]{7}{*}{1.5} & -1.5 & 0.53 & 1.01 & 10.8 & 17.3 & 0.95 & 0.94 \\
\hline & -1.0 & 12.4 & 9.83 & 28.8 & 28.2 & 0.86 & 0.91 \\
\hline & -0.5 & 2.57 & 3.45 & 59.1 & 35.9 & 0.83 & 0.91 \\
\hline & 0.0 & 210 & - & 22.5 & 24.1 & 0.91 & 0.91 \\
\hline & +0.5 & - & 153 & 40.7 & 19.4 & 0.88 & 0.93 \\
\hline & +1.0 & 43.4 & 64.7 & 21.8 & 15.1 & 0.90 & 0.94 \\
\hline & +1.5 & 5.59 & 0.41 & 8.24 & 13.2 & 0.95 & 0.94 \\
\hline \multirow[t]{7}{*}{15} & -1.5 & 0.24 & 2.76 & 17.2 & 90.7 & 0.88 & 0.83 \\
\hline & -1.0 & 7.62 & 4.45 & 26.3 & 88.7 & 0.84 & 0.81 \\
\hline & -0.5 & 8.03 & 3.78 & 24.9 & 70.6 & 0.86 & 0.83 \\
\hline & 0.0 & 186 & 47.3 & 23.3 & 26.4 & 0.84 & 0.88 \\
\hline & +0.5 & 400 & 77.6 & 20.2 & 22.8 & 0.86 & 0.89 \\
\hline & +1.0 & 76.5 & 70.7 & 19.2 & 19.2 & 0.87 & 0.91 \\
\hline & +1.5 & 3.66 & 0.22 & 11.3 & 25.7 & 0.92 & 0.94 \\
\hline \multirow[t]{7}{*}{140} & -1.5 & 0.25 & 0.51 & 69.1 & 33.9 & 0.70 & 0.81 \\
\hline & -1.0 & 6.75 & 8.33 & 25.7 & 34.0 & 0.80 & 0.83 \\
\hline & -0.5 & 20.9 & 23.7 & 35.6 & 38.7 & 0.79 & 0.83 \\
\hline & 0.0 & - & - & 41.7 & 38.2 & 0.78 & 0.81 \\
\hline & +0.5 & - & - & 31.9 & 27.3 & 0.81 & 0.85 \\
\hline & +1.0 & 119 & 79.1 & 21.4 & 20.6 & 0.83 & 0.87 \\
\hline & +1.5 & 1.24 & 0.97 & 26.5 & 25.3 & 0.78 & 0.83 \\
\hline
\end{tabular}

Before pre-treatment, characteristic peaks for hexacyanoferrate(II) in cyclic voltammograms at electrodes of $1.5 \Omega$, as well as of $15 \Omega$, showed peaks that were less well-defined than those in Fig. 4 , indicative of a slower electron transfer reaction. Estimations of $k_{0}$ gave values between (1.2 and 1.6) $\times 10^{-3} \mathrm{~cm} \mathrm{~s}^{-1}$ for $1.5 \Omega$ electrodes, and $3.0 \times 10^{-3} \mathrm{~cm} \mathrm{~s}^{-1}$ for $15 \Omega$ electrodes. In the case of $15 \Omega$, there was no improvement in the cyclic voltammograms after electrode pre-treatment. Unfortunately, electrodes con-
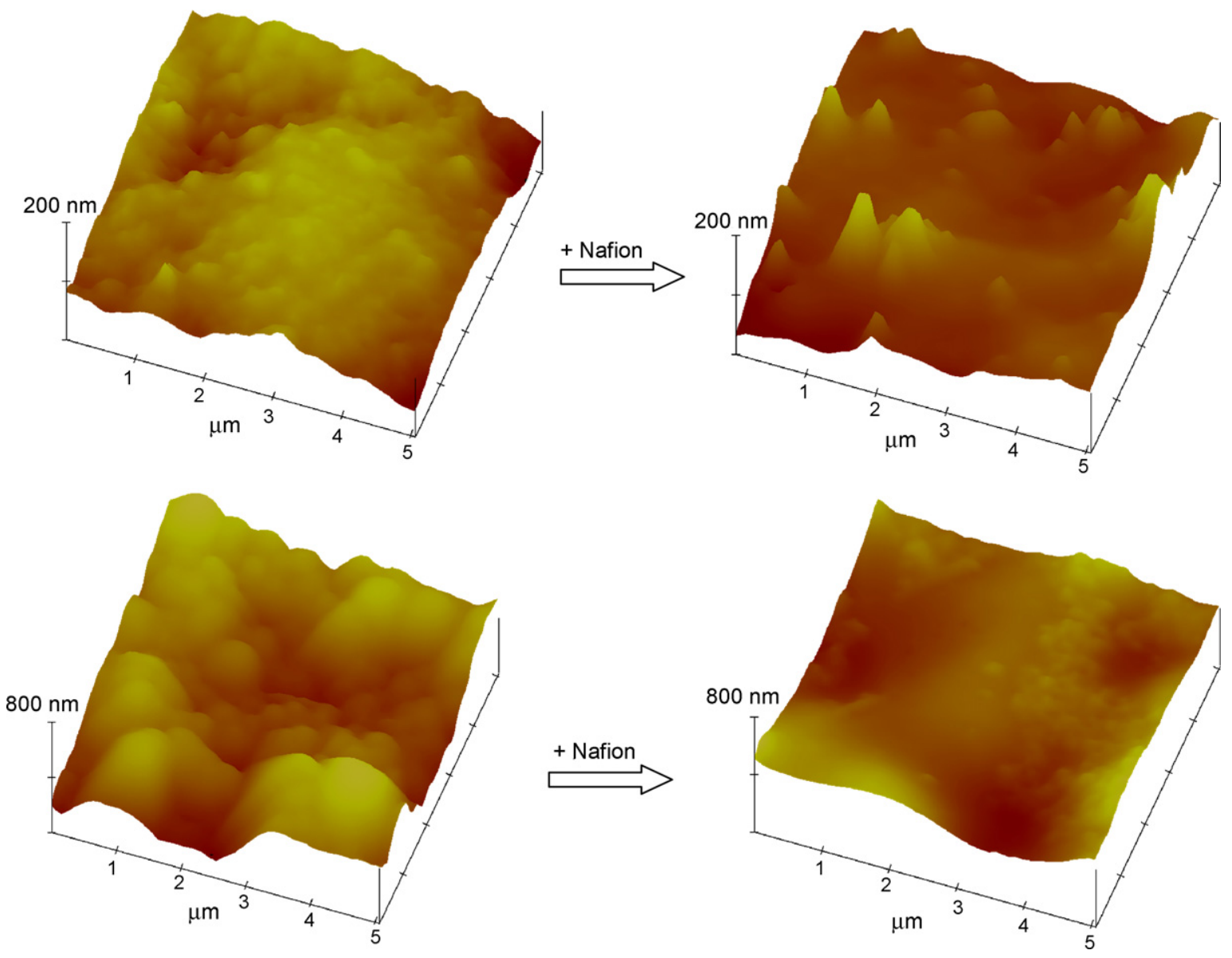

Fig. 6. 3D AFM topographical images of carbon film electrodes of $1.5 \Omega$ (upper) and $140 \Omega$ (bottom) nominal resistances.

Please cite this article in press as: C. Gouveia-Caridade et al., Electrochemical, morphological and microstructural characterization of carbon film resistor electrodes for application in electrochemical sensors, Appl. Surf. Sci. (2008), doi:10.1016/j.apsusc.2008.03.180 
structed from resistors of value greater than $15 \Omega$ nominal resistance led to poorly defined voltammograms, so that it is not possible to extract any information from them concerning the kinetics of the $\left[\mathrm{Fe}(\mathrm{CN})_{6}\right]^{3-/ 4-}$ redox couple.

\subsubsection{Electrochemical impedance spectroscopy}

Electrochemical impedance experiments were carried out for electrodes from resistors of $1.5,15$ and $140 \Omega$ in the two different electrolytes at values of applied potential that cover the whole available potential range, i.e. between -1.5 and $+1.5 \mathrm{~V}$. Spectra at -1.0 and $-0.5 \mathrm{~V}$ are shown in Fig. 5.

In general, higher impedance values were obtained in phosphate buffer saline than in acetate buffer solution. The shape of impedance spectra obtained at negative potentials for electrodes of 1.5 and $15 \Omega$ was essentially the same, although larger impedance values were found with $1.5 \Omega$ resistors. In this case, the values were similar to those of the $2 \Omega$ resistors reported in $[3,4]$, except at $-1.0 \mathrm{~V}$, where a semi-circle obtained at $2 \Omega$ electrodes was due to hydrogen evolution occurring at the electrode surface. This different behaviour can be attributed to the distinct pre-treatment used (perchloric acid for the $2 \Omega$ resistor electrodes), producing different surface functionalities.

At 0.0 and $+0.5 \mathrm{~V}$, in the two electrolytes studied, the behaviour of all types of electrode was characteristic of a non-ideal capacitor, as at $2 \Omega$ electrodes $[3,4]$. Above $+0.5 \mathrm{~V}$, oxygen evolution was responsible for the semi-circular shape and lowest magnitude impedance values.

Fitting of the higher frequency part of the spectra was done using an equivalent electrical circuit, which comprised the cell resistance, $R_{\Omega}$, in series with a parallel $R C P E$ combination. The constant phase element, $\mathrm{CPE}$, was necessary due to the depressed semi-circle character of the responses, that can be attributed to the non-homogeneous surface, and is modelled as a non-ideal capacitor of capacitance $C$ and roughness exponent $\alpha$, according to $\mathrm{CPE}=-1 / C(\mathrm{i} \omega)^{\alpha}$, where an $\alpha$ value of 1 represents a perfectly smooth surface. This circuit was the same as used in [3,4] for carbon film electrodes at frequencies above $1 \mathrm{~Hz}$. Below this frequency, reproducibility between experiments was lower and the form of the spectrum also depended on the applied potential. In the chosen frequency range, the errors in the fitting of the experimental values to the equivalent circuit varied between 1 and $10 \%$ for $R_{1}, 0.8$ and $3 \%$ for $C$, and less than $1 \%$ for $\alpha_{1}$. Results obtained are shown in Table 2.

The cell resistance, $R_{\Omega}$, was higher in acetate buffer solution and increases with the nominal resistance of the electrode, being $42 \pm 1,47 \pm 1$ and $61 \pm 2 \Omega \mathrm{cm}^{2}$ for seven spectra at $1.5,15$ and $140 \Omega$ electrodes, respectively. In phosphate buffer solution the values, measured for 7 spectra, were $7.6 \pm 0.2 \Omega \mathrm{cm}^{2}$ for $1.5 \Omega$, $9.2 \pm 0.3 \Omega \mathrm{cm}^{2}$ for $15 \Omega$, and $26 \pm 1 \Omega \mathrm{cm}^{2}$ for $140 \Omega$ resistors.
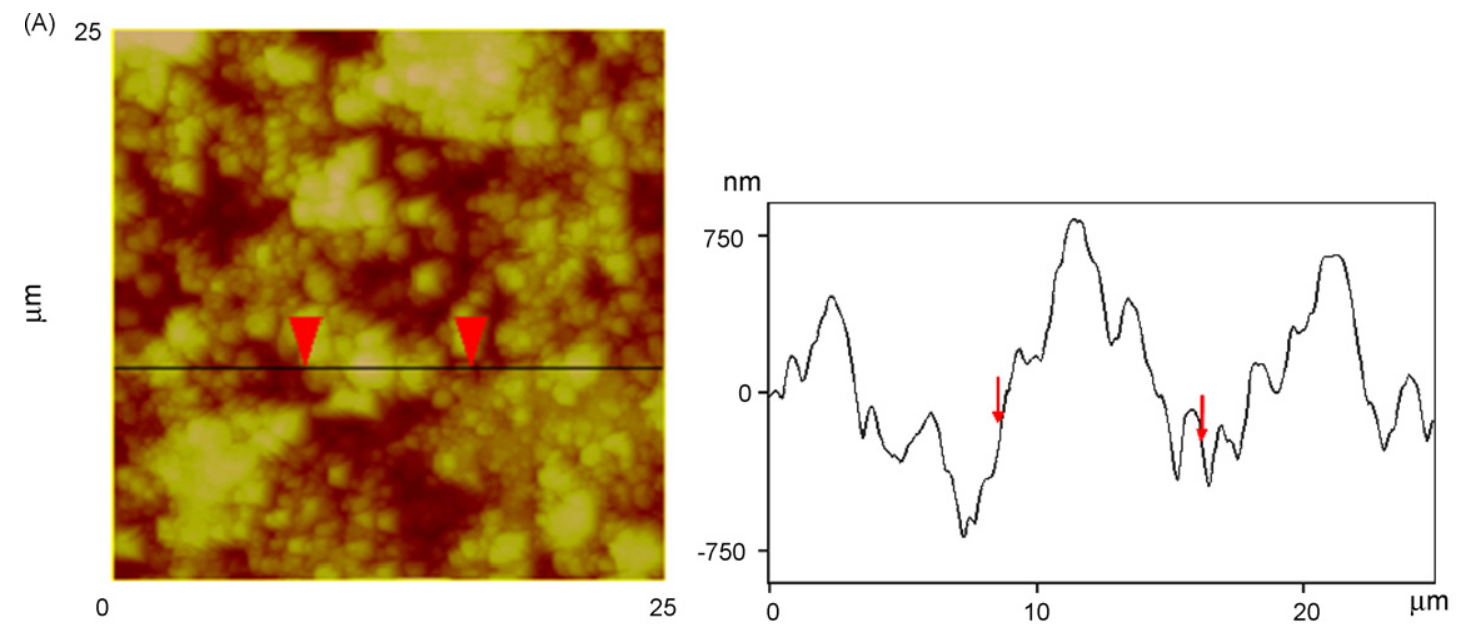

$\mu \mathrm{m}$
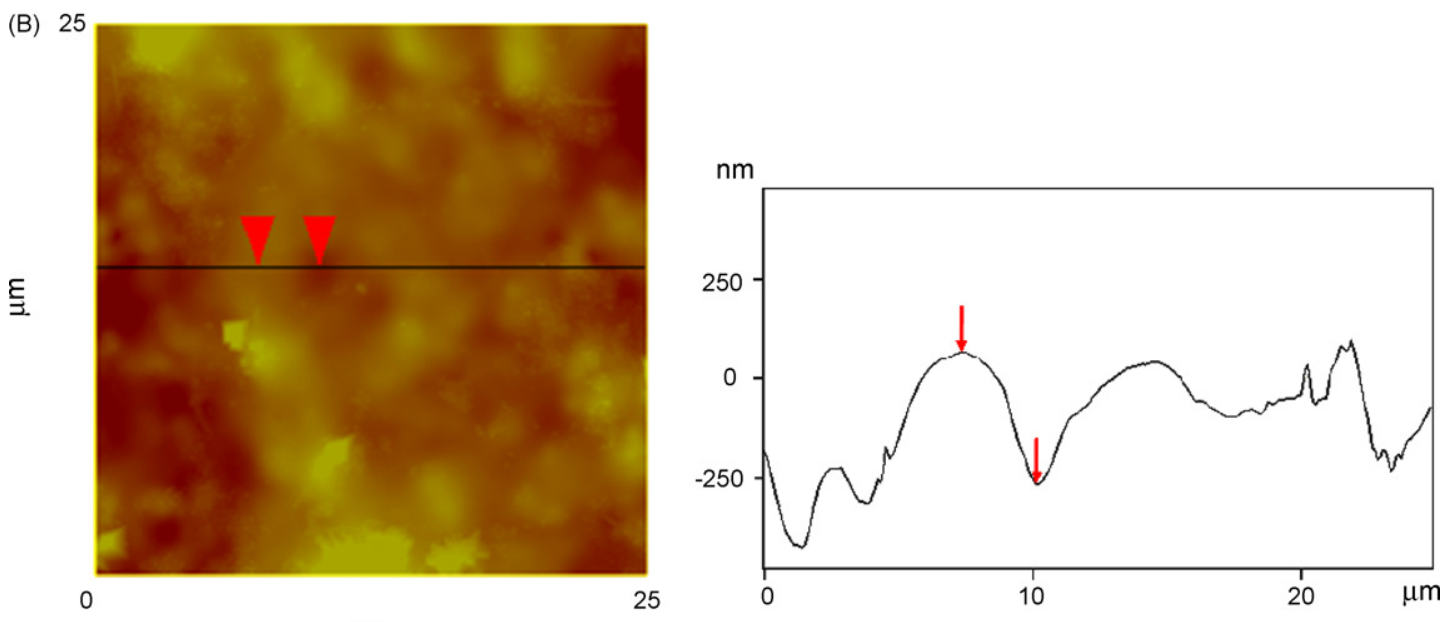

$\mu \mathrm{m}$

Fig. 7. Section analysis of 2D AFM images of $140 \Omega$ electrodes (A) without and (B) with Nafion coating. 
In both acetate buffer and PBS solution, at negative applied potentials the general trend is higher charge transfer resistance and higher capacitance at $1.5 \Omega$ resistor electrodes compared with 15 and $140 \Omega$ resistors. However, at $-0.5 \mathrm{~V}$ a higher capacitance and lower $R_{1}$ was obtained, which can be due to easier reduction of surface oxide and of dissolved oxygen at these films, which are thicker than those of the other two resistors of higher value nominal resistance. As mentioned previously, all electrodes were fabricated by pyrolysis of methane on the surface of a ceramic cylinder, the film thickness being inversely related to the nominal resistance of the electrode.

At positive applied potentials, when increasing the potential the tendency observed is not so clear, although at $+0.5 \mathrm{~V}$ the behaviour is similar to that observed at negative potentials, i.e. higher charge transfer resistance and higher interfacial capacitance with the $1.5 \Omega$ resistor electrodes. Another interesting observation is that in PBS solution, the $15 \Omega$ resistor showed the highest capacitances, and at negative applied potentials the charge transfer is lower suggesting that the charge transfer reaction should occur more easily.
(A)

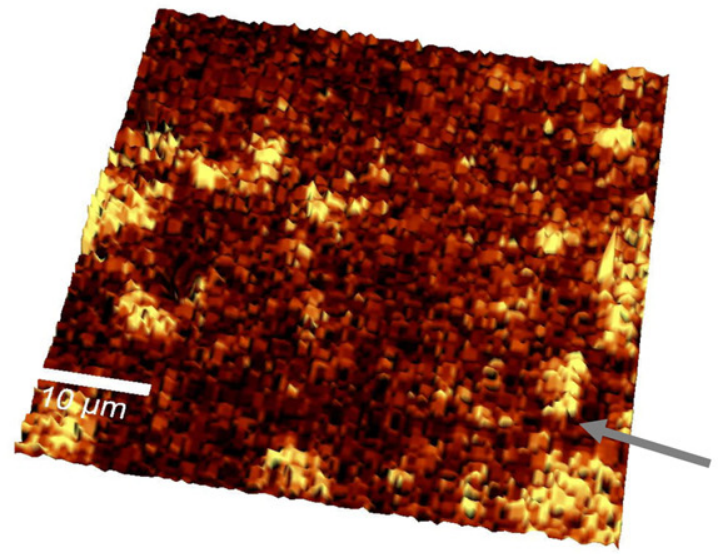

(B)

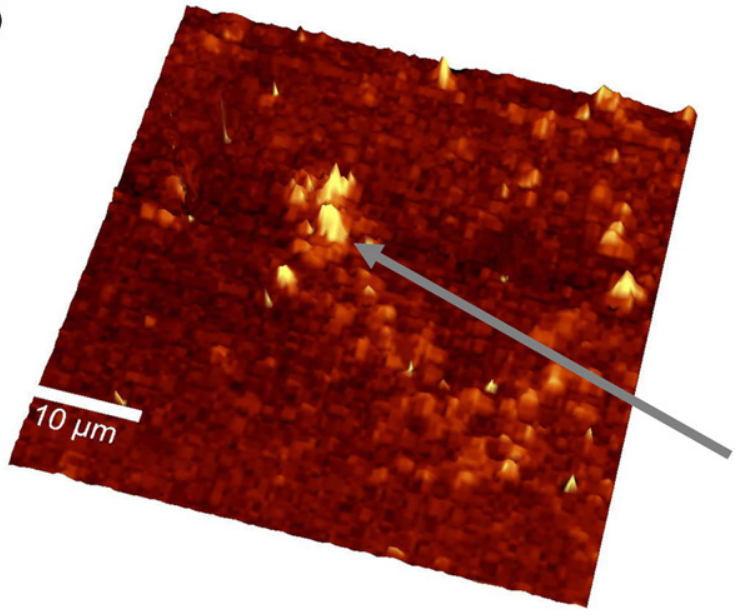

(C)

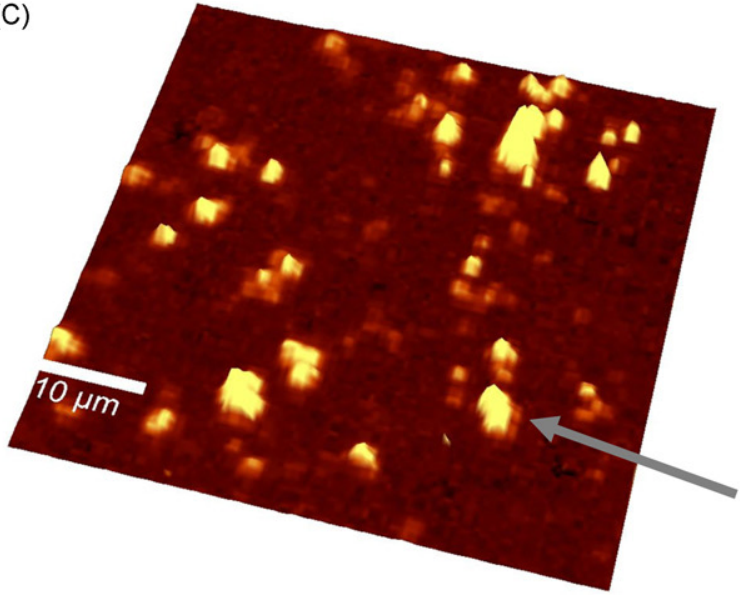

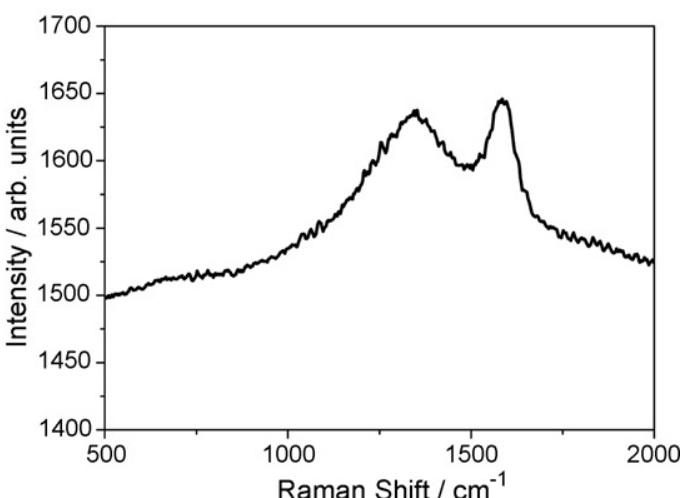
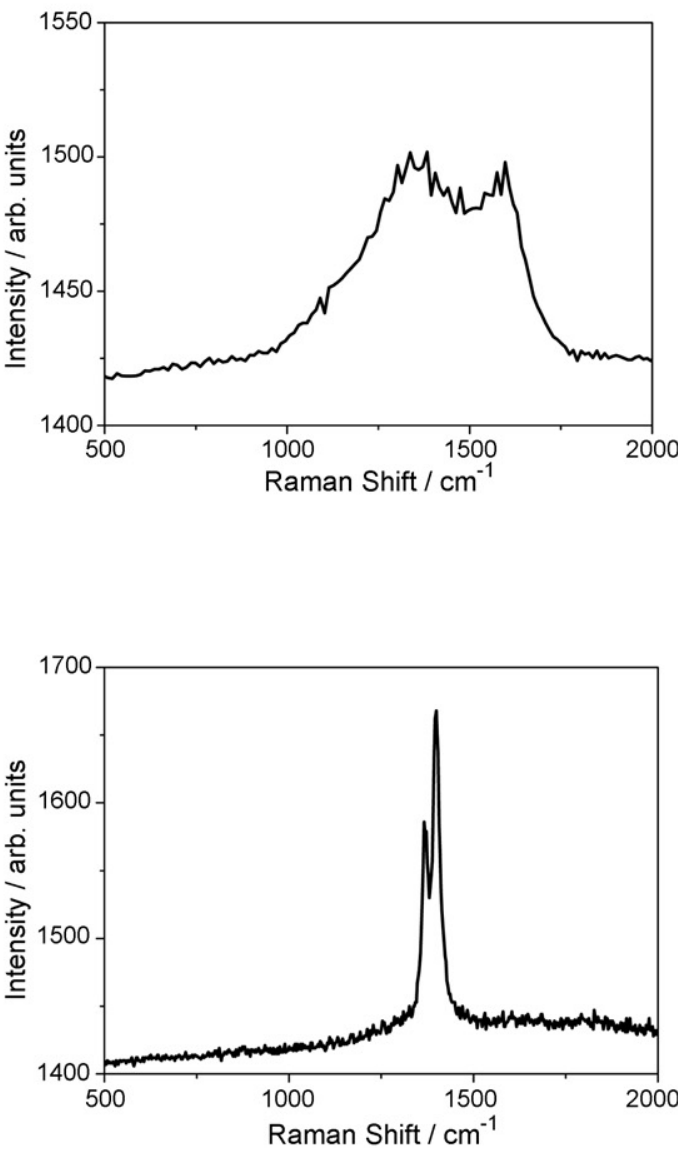

Fig. 8. Confocal Raman spectra of carbon film electrodes of: (A) $1.5 \Omega$, (B) $2.0 \Omega$ and (C) $140 \Omega$ nominal resistances. 
At the limits of applied potential $(-1.5$ and $+1.5 \mathrm{~V})$ the lowest values of charge transfer resistance were due to some evolution of hydrogen and oxygen, respectively.

The values of the exponent $\alpha$ were higher in phosphate buffer solution, and decreased with increasing nominal resistance of the electrodes, revealing the smoother surface of the lower resistance electrodes.

Plots of $-Z^{\prime \prime}$ vs $\lg f$ (not shown) together with the complex plane plots lead to the following conclusions:

- The shape of the spectra is not altered by the solution used.

- At $-0.5 \mathrm{~V}$ the peak in plots in $-Z^{\prime \prime}$ vs $\lg f$ shifts to lower values with increasing resistance, which can be attributed to a more difficult reduction of surface oxide and of dissolved oxygen at electrodes of $140 \Omega$.

- At positive applied potentials there is no evidence of charge transfer processes. The same occurs at $-1.0 \mathrm{~V}$ for 1.5 and $15 \Omega$ electrodes. However, with electrodes of $140 \Omega$ at $-1.0 \mathrm{~V}$ at frequencies below $1 \mathrm{~Hz}$ another feature appears which can be related to the occurrence of another process, probably due to the presence of non-conductive materials introduced in the carbon film production to increase its nominal resistance.

\subsection{Surface analysis}

\subsubsection{Atomic Force Microscopy}

Contact-mode AFM was used to examine the local morphology of the carbon film resistors of various nominal resistances without pre-treatment. Fig. 6 shows 3D images obtained for 1.5 and $140 \Omega$ resistors before and after coating with a Nafion film. AFM images of 2 and $15 \Omega$ were similar to those obtained with $1.5 \Omega$ resistors, and $2.0 \mathrm{k} \Omega$ was similar to that obtained with $140 \Omega$. The AFM images evidence the nanoscale morphology of the carbon film.

Profile and roughness analysis were carried out in images of $25 \mu \mathrm{m} \times 25 \mu \mathrm{m}$, revealing some differences in morphological parameters with the type of resistor. In general, the mean roughness, $R_{\mathrm{a}}$, defined as the average absolute deviation of the roughness irregularities from the mean line over one sampling length [19]

$R_{\mathrm{a}}=\frac{1}{n} \sum_{i=1}^{n}\left|y_{i}\right|$

increases with increasing electrode resistance, with typical values of $\sim 88 \mathrm{~nm}$ for $1.5 \Omega$ resistors and $\sim 270 \mathrm{~nm}$ for those of $140 \Omega$. Although the same trends in $R_{\mathrm{a}}$ were observed for Nafion-coated resistors, typical values in this case were $\sim 86 \mathrm{~nm}$ for $1.5 \Omega$ and $\sim 147 \mathrm{~nm}$ for $140 \Omega$ coated resistors. Moreover, the root mean square roughness, $R_{\mathrm{q}}$, defined as the standard deviation of the distribution of surface heights [19],

$R_{\mathrm{q}}=\sqrt{\frac{1}{n} \sum_{i=1}^{n} y_{i}^{2}}$

which is more sensitive than $R_{\mathrm{a}}$ to large deviations from the mean line, follows the same trends as $R_{\mathrm{a}}$, with a more perceptible decrease in values obtained after coating surfaces with Nafion. For example, resistors of $140 \Omega$ had $R_{\mathrm{q}} \sim 340 \mathrm{~nm}\left(R_{\mathrm{a}} \sim 270 \mathrm{~nm}\right)$ without Nafion and $R_{\mathrm{q}} \sim 200 \mathrm{~nm}\left(R_{\mathrm{a}} \sim 147 \mathrm{~nm}\right)$ with the Nafion coating.

The section profile obtained with a $140 \Omega$ resistor is illustrated in Fig. 7. It can be seen that with Nafion the maximum height decreases. The same behaviour was observed in the other resistors studied. The images were recorded in different areas of the electrodes and the same trends and observations made.
The main important conclusion from the AFM images is that with a decrease in electrode resistance, the carbon film roughness decreases and the surface uniformity increases. The electrical resistance is inversely proportional to the film thickness (assuming constant resistivity), so that whereas the carbon film can be expected to follow the contours of the ceramic cylinder substrate initially, as the film grows thicker its influence will be less. The roughness parameters calculated from the AFM images are in total agreement with the roughness exponent, $\alpha$, from EIS experiments: for $1.5 \Omega$ electrodes, $\alpha$ is closer to 1 and the roughness calculated from AFM is lower due to the smoother surface. The flatter and thicker carbon film is related to better electrochemical characteristics.

\subsubsection{Confocal Raman spectroscopy and X-ray diffraction}

3.2.2.1. Carbon film resistors. Raman shifts and scattering in 1.5, 2.0 and $140 \Omega$ carbon film resistors without pre-treatment are shown in Fig. 8. The two bands obtained for all of these resistors are consistent with a graphitic structure: $G$ band at around $1594 \mathrm{~cm}^{-1}$ and $D$ band at around $1345 \mathrm{~cm}^{-1}$. The same bands were obtained for resistors of $15 \Omega$ and $2.0 \mathrm{k} \Omega$.

Raman spectra are usually discussed in the context of diamond versus graphite as carbon films are composed of short distance ordered $\mathrm{sp}^{3}$ and $\mathrm{sp}^{2}$ bonds. Differences between diamond films and these carbon films can be ascribed to surface microstructure. Diamond films are made by pyrolysis of dilute mixtures of a hydrocarbon gas in hydrogen using energy-assisted chemical vapour deposition - hot-filament or microwave discharge - which leads to a $\mathrm{sp}^{3}$-hybridized structure. The carbon film resistor preparation here uses heated unreactive nitrogen as the main gas with heating which precludes the formation of methyl radicals and atomic hydrogen and leads to a more reactive $\mathrm{sp}^{2}$-hybridized graphite microstructure $[2,20,21]$.

Diamond has a single Raman active mode at $1332 \mathrm{~cm}^{-1}$, and another Raman line at $1575 \mathrm{~cm}^{-1}$, the "G" peak, reflecting the vibration mode of perfect graphite [14,22,23]. Multi-crystalline graphite also shows an additional sharp "D" peak at $1355 \mathrm{~cm}^{-1}$, reflecting the mode for disorder [14,24]. In HOPG the creation of disorder, and therefore the creation of $\mathrm{D}$ band, attributed to the presence of edge graphite microcrystallites [25-27], by laser activation or electrochemical pre-treatment, promotes the nucleation and growth of defect sites.

$\mathrm{X}$-ray diffraction was performed in order to obtain further information on and confirmation of the structure of the carbon. The

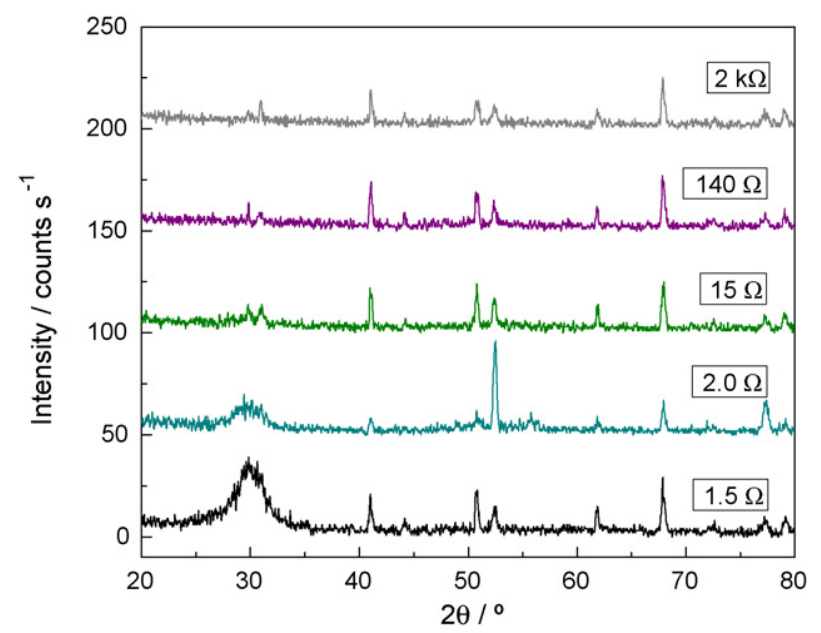

Fig. 9. XRD patterns of carbon film electrodes of $1.5 \Omega$ to $2.0 \mathrm{k} \Omega$ nominal resistance. 
(A)
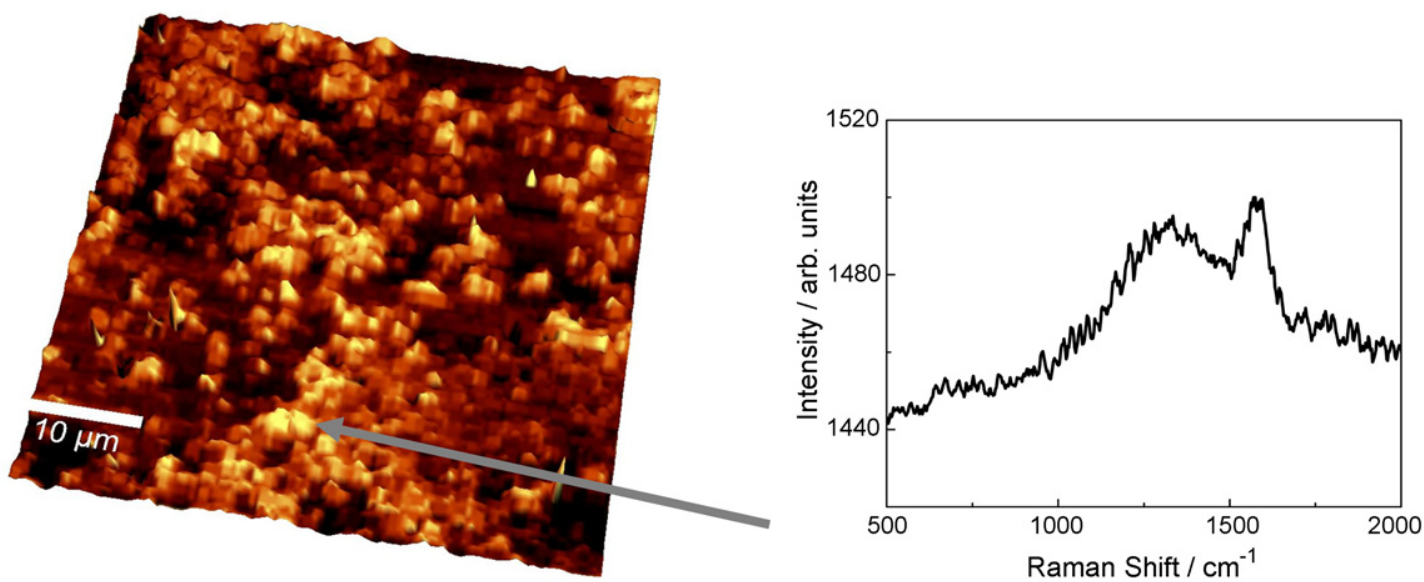

(B)
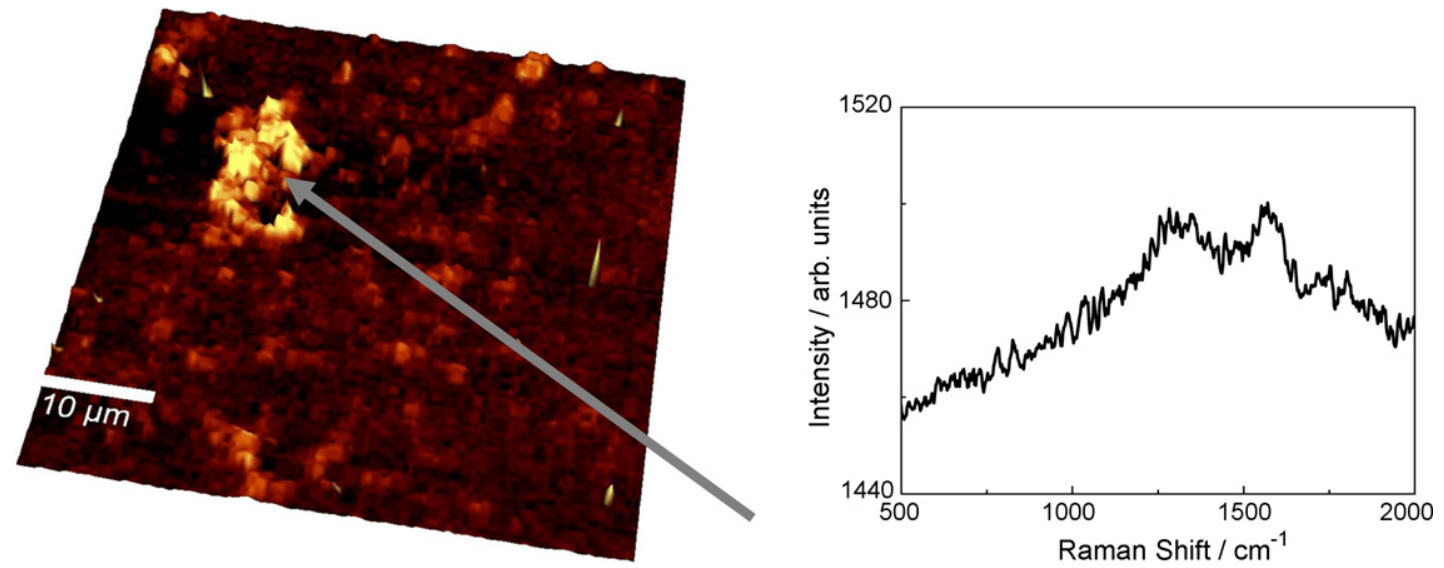

Fig. 10. Confocal Raman spectra of Nafion-coated carbon film electrodes of (A) $1.5 \Omega$ and (B) $2.0 \Omega$ nominal resistances.

diffraction line at $\sim 31.0^{\circ}$ for $2 \theta$ in the XRD diffraction patterns in Fig. 9 for all types of carbon film electrode studied also indicate the existence of the graphitic structure in the carbon films.

3.2.2.2. Nafion-coated carbon film resistors. Carbon films of various resistances were modified with a Nafion film as described in Section 2. Nafion-coated carbon film electrodes are important for application as trace heavy metal sensors in natural media and were previously electrochemically characterized [4]. The modified electrodes were analyzed by Confocal Raman spectroscopy in order to throw light on whether the Nafion film changes the Raman characteristics of the carbon film electrodes. If this happened it would suggest a strong chemical interaction between the Nafion film and the carbon film surface.

Fig. 10 shows the Raman shift and scattering of Nafion-coated 1.5 and $2.0 \Omega$ resistors. Spectra similar to those in Fig. 8 were obtained for the other resistors modified with Nafion polymer. Comparing Figs. 8 and 10 it is possible to conclude that the Nafion film does not change the Raman spectral profile of carbon film electrodes.

\section{Conclusions}

The characteristics of carbon film electrodes made from carbon film resistors of various nominal resistances were investigated to clarify their potential use in electrochemical sensors.

Resistors of $1.5 \Omega$ exhibited similar electrochemical properties, after electrochemical pre-treatment, to those obtained with previously used $2 \Omega$ resistors. Good potential windows were obtained at 15 and $140 \Omega$ resistor electrodes but exhibited insufficiently low currents for oxidation and reduction of electro- active species, as well as slow electrode kinetics, to be applied in sensors. Electrodes from $2.0 \mathrm{k} \Omega$ resistors show poor cyclic voltammetric profiles even after pre-treatment. EIS data clearly showed differences in the interfacial behaviour of electrodes.

AFM shows that with a decrease in electrode resistance, the homogeneity and smoothness of the carbon film increases, which can be related to better electrochemical characteristics. Confocal Raman spectroscopy at all electrodes reveals a G band at $1594 \mathrm{~cm}^{-1}$ and a D band at around $1345 \mathrm{~cm}^{-1}$ consistent with a graphitic structure. No alterations to Raman spectra were visualized with Nafion-coated carbon film electrodes. XRD diffraction patterns are consistent with a graphitic structure in all type of carbon film electrode studied and confirm the Raman analysis.

\section{Acknowledgements}

Financial support from Fundação para a Ciência e Tecnologia (FCT), Portugal, PTDC/QUI/65255/2006, POCI 2010 (co-financed by the European Community Fund FEDER), GRICES/DAAD Portugal/ Germany bilateral agreement in science and technology and ICEMS (Research Unit 103), is gratefully acknowledged. The authors are grateful to O. Teschke for Scanning Near-Field Optical Microscope measurements, and to L.O. Bonugli for technical assistance, and to J.P. Dias for the X-ray diffraction measurements. CGC thanks FCT for a Ph.D. grant (SFRH/BD/18659/2004).

\section{References}

[1] C.M.A. Brett, Pure Appl. Chem. 73 (2001) 1969

[2] C.M.A. Brett, L. Angnes, H.D. Liess, Electroanalysis 13 (2001) 765. 
[3] O.M.S. Filipe, C.M.A. Brett, Electroanalysis 16 (2004) 994.

[4] C. Gouveia-Caridade, C.M.A. Brett, Electroanalysis 17 (2005) 549.

[5] O.M.S. Filipe, C.M.A. Brett, Talanta 61 (2003) 643.

[6] R. Pauliukaite, C.M.A. Brett, Electroanalysis 17 (2005) 1354.

[7] R. Pauliukaite, M. Florescu, C.M.A. Brett, J. Solid State Electrochem. 9 (2005) 354.

[8] R. Pauliukaite, M.E. Ghica, C.M.A. Brett, Anal. Bioanal. Chem. 381 (2005) 972.

[9] C. Gouveia-Caridade, C.M.A. Brett, J. Electroanal. Chem. 192 (2006) 113.

[10] M.E. Ghica, C.M.A. Brett, Anal. Lett. 38 (2005) 907

[11] M. Florescu, C.M.A. Brett, Talanta 65 (2005) 306.

[12] R. Pauliukaite, A.M. Chiorcea-Paquim, A.M. Oliveira-Brett, C.M.A. Brett, Electrochim. Acta 52 (2006) 1

[13] M.E. Ghica, C.M.A. Brett, Electroanalysis 18 (2006) 748

[14] P.K. Chu, L.H. Li, Mater. Chem. Phys. 96 (2006) 253.

[15] W. Hoheisel, W. Jacobsen, B. Lüttge, W. Weiner, Macromol. Mater. Eng. 286 (2001) 663.
[16] C. Gouveia-Caridade, R. Pauliukaite, C.M.A. Brett, Electroanalysis 18 (2006) 854

[17] R.S. Nicholson, Anal. Chem. 37 (1965) 1351.

[18] H. Maleki, C.D. Cojocaru, C.M.A. Brett, G.M. Jenkins, J.R. Selman, J. Electrochem. Soc. 145 (1998) 721

[19] E.S. Gadelmawla, M.M. Koura, T.M.A. Maksoud, I.M. Elewa, H.H. Soliman, J. Mater Process. Technol. 123 (2002) 133.

[20] A. Argoitia, J.C. Angus, J.S. Ma, L. Wang, P. Pirouz, W.R.L. Lambrecht, J. Mater. Res. 9 (1994) 1849.

[21] H.B. Martin, A. Argoitia, J.C. Angus, U. Landau, J. Electrochem. Soc. 146 (1999) 2959.

[22] F. Tuinstra, J.L. Koenig, J. Chem. Phys. 53 (1970) 1126.

[23] J. Robertson, Mater. Sci. Eng. R 37 (2002) 129.

[24] J. Robertson, Surf. Coat. Technol. 50 (1992) 185

[25] R.J. Bowling, R.T. Packard, R.L. McCreery, J. Am. Chem. Soc. 111 (1989) 1217.

[26] R.J. Rice, R.L. McCreery, Anal. Chem. 61 (1989) 1637.

[27] Y.W. Alsmeyer, R.L. McCreery, Langmuir 7 (1991) 2370.

Please cite this article in press as: C. Gouveia-Caridade et al., Electrochemical, morphological and microstructural characterization of carbon film resistor electrodes for application in electrochemical sensors, Appl. Surf. Sci. (2008), doi:10.1016/j.apsusc.2008.03.180 